\title{
Floquet engineering of gapped 2D materials
}

\author{
(C) O.V. Kibis ${ }^{1}$, K. Dini ${ }^{2}$, I.V. Iorsh ${ }^{3}$, I.A. Shelykh $h^{2,3}$ \\ ${ }^{1}$ Department of Applied and Theoretical Physics, Novosibirsk State Technical University, \\ 630073 Novosibirsk, Russia \\ ${ }^{2}$ Science Institute, University of Iceland, Dunhagi 3, \\ IS-107, Reykjavik, Iceland \\ ${ }^{3}$ ITMO University, \\ 197101 St. Petersburg, Russia \\ E-mail: oleg.kibis@nstu.ru
}

It is demonstrated theoretically that the interaction of gapped 2D materials (gapped graphene and transition metal dichalchogenide monolayers) with a strong high- crucially changes the band structure of the materials. As a consequence, the renormalized band structure of the materials drastically depends on the field polarization. Particularly, a linearly polarized dressing field always decreases band gaps, whereas a circularly polarized field breaks the equivalence of band valleys in different points of the Brillouin zone and can both increase and decrease corresponding band gaps. It is shown also that a dressing field can turn both the band gaps and the spin splitting of the bands into zero. As a result, the dressing field can serve as an effective tool to control spin and valley properties of the materials in various optoelectronic applications.

\section{Acknowledgements}

The work was partially supported by RISE Program (project CoExAN), FP7 ITN Program (project NOTEDEV), Russian Foundation for Basic Research (project 17-02-00053), Rannis project 163082-051, and Ministry of Education and Science of Russian Federation (projects 3.4573.2017/6.7, 3.2614.2017/4.6, 3.1365.2017/4.6, 3.8884.2017/8.9 and 14.Y26.31.0015) 